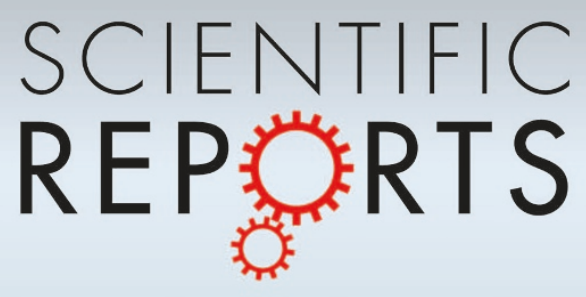

OPEN

SUBJECT AREAS:

INFORMATION STORAGE

APPLIED PHYSICS

MAGNETIC PROPERTIES AND MATERIALS

MAGNETIC DEVICES

Received

24 June 2013

Accepted

30 September 2013

Published

16 October 2013

Correspondence and requests for materials should be addressed to

X.J.X. (xxjun@wzu. edu.cn)

\section{How do spin waves pass through a bend?}

\author{
Xiangjun Xing' ', Yongli Yu' , Shuwei Li \& Xiaohong Huang'
}

${ }^{1}$ College of Physics and Electronic Information Engineering, Wenzhou University, Wenzhou 325035, China, ${ }^{2}$ State Key Laboratory of Optoelectronic Materials and Technologies, School of Physics and Engineering, Sun Yat-sen University, Guangzhou 510275, China.

Spin-wave devices hold great promise to be used in future information processing. Manipulation of spin-wave propagation inside the submicrometer waveguides is at the core of promoting the practical application of these devices. Just as in today's silicon-based chips, bending of the building blocks cannot be avoided in real spin-wave circuits. Here, we examine spin-wave transport in bended magnonic waveguides at the submicron scale using micromagnetic simulations. It is seen that the impact of the bend is relevant to the frequency of the passing spin wave. At the lowest frequencies, the spin wave continuously follows the waveguide in the propagation process. At the higher frequencies, however the bend acts as a mode converter for the passing spin wave, causing zigzag-like propagation path formed in the waveguide behind the bend. Additionally, we demonstrate a logic-NOT gate based on such a waveguide, which could be combined to perform logic-NAND operation.

pin-wave devices ${ }^{1-5}$ are deemed as the most promising candidates for the present electronic ones ${ }^{6}$. MachZehnder type spin-wave logic gates are made of long magnonic waveguides, where spin-wave propagation is manipulated to implement logic operations ${ }^{1-4}$. Real spin-wave chips will inevitably integrate numerous functional units to do powerful computation. Bended components will have to be used to save space ${ }^{7}$. Consequently, spin-wave propagation in bended magnonic waveguides should be addressed.

Bance et al. reported the transmission of backward-volume (BV) spin-wave packets through a $90^{\circ}$ circular bend without losses ${ }^{8}$; nevertheless they did not identify the mode distribution of these spin waves, which is essential to understand the spin-wave propagation inside the bend. Quite recently, Dvornik et al. demonstrated the fact of the BV spin waves to follow a curved waveguide without noticeable losses at the bend ${ }^{7}$, and thus claimed that the spin waves adapt the bend with their wave vectors parallel to the local magnetization. By contrast, Clausen et al. observed the multimode excitation of Damon-Eshbach (DE) spin waves in the post-skew arm of a skewed waveguide ${ }^{9}$. Considering the similarity between the bended and skewed waveguides, the results reported by Dvornik et al. should be a special instance of spin-wave transport in bended waveguides, since only a single frequency is inspected there ${ }^{7}$. Vogt et al. realized efficient transmission of the DE spin waves through an analogous bend by introducing a novel method to align the magnetization inside the bend ${ }^{10}$, but they did not measure the mode distribution of the spin waves past the bend. To be used in nanoscale logic circuits, the DE spin waves are inferior to the BV ones, because they require an extra magnet to align the magnetization ${ }^{7}$.

In Mach-Zehnder type logical gates, an external local field is frequently used to tailor the parameters of the propagating spin waves, as done in Refs. 2-4. Unfortunately, Vasiliev et al. ${ }^{11}$ pointed out that the spin-wave logic devices using an external field to control the spin-wave propagation would face intrinsic limitations, with respect to the operation efficiency by the applied field, as the waveguide is further downscaled. Therefore, it is necessary to find fresh means of constituting nanoscale interferometric logic gates. Hertel et al. ${ }^{1}$ explored a novel route to topologically induce a phase shift of spin waves by introducing a domain wall, and utilized it to construct a MachZehnder type logic-XOR gate. However, it is not easy to precisely manipulate a domain wall with the desirable structure in nanocale magnetic waveguides ${ }^{2}$.

In this Letter, we study the propagation of spin waves in bended magnonic waveguides with the magnetization aligned along the waveguide. We find that the spin waves do not always adapt to the waveguide, and only at some frequencies can the spin waves follow the waveguide without significant losses. At the other frequencies, antisymmetric width modes ${ }^{9,12}$ are excited inside the bend once the symmetric modes emitted from the antenna pass through, and then multimode spin waves superpose in the horizontal arm of the waveguide, causing a spatial beating of these modes ${ }^{13}$. The beaten spin waves can be injected into two separate waveguide branches, where the injected beams have a phase shift of $\sim \pi$ at some specific frequencies. Further, we demonstrate an interferometric logic-NOT gate of the Mach-Zehnder type, whose logical input is encoded by the frequency of the carrying spin wave itself. 


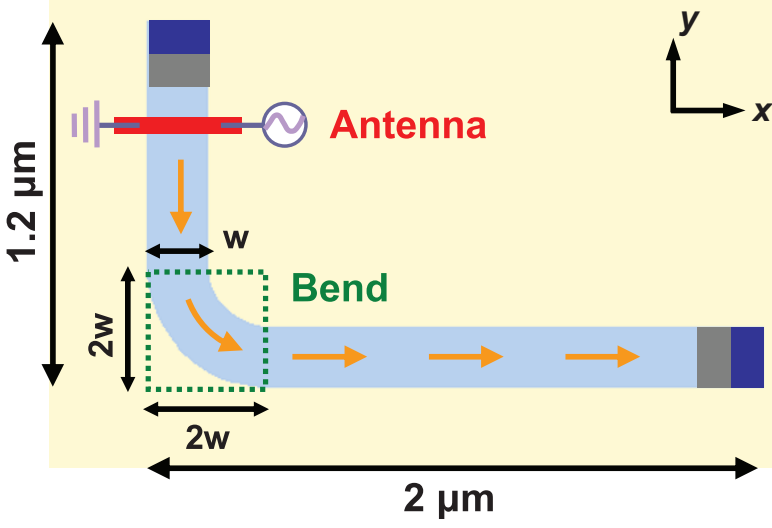

Figure $1 \mid$ Setup for spin-wave excitation and propagation in a bended magnetic waveguide. The inner and outer radii of the bend are equal to $w$ and $2 w$, respectively, where $w$ is the width of the waveguide. In the present work, $w=100$ and $200 \mathrm{~nm}$, and the thickness of the waveguide is $5 \mathrm{~nm}$. The vertical and horizontal dimensions of the waveguide are 1.2 and $2 \mu \mathrm{m}$, respectively. The gray and blue regions at the end of the waveguide form an absorbing boundary (Ref. 8). No bias field is imposed. The microstrip antenna is a source for traveling spin waves. The antenna is fixed at such a position that the distance between its centre and the entry of the bend is $500 \mathrm{~nm}$.

\section{Results}

The sketch of the present study is shown in Fig. 1. The magnonic waveguide is a two-dimensional submicrometer magnetic strip including a $90^{\circ}$ circular bend. The size of the target system is marked in the figure. In this work, two kinds of waveguides with the widths equal to 200 and $100 \mathrm{~nm}$ are considered (i.e. $w=100$ and $200 \mathrm{~nm}$ ). No bias field is applied to the waveguide, and thus the magnetization is aligned along the waveguide by the shape anisotropy, resulting in the BV geometry ${ }^{14}$ for spin-wave propagation. A microstrip antenna for spin-wave excitation is placed in front of the bended section of the waveguide. A radio-frenquency field in the form of $h_{\mathrm{ac}}(t)=$ $H_{0} \cdot \sin (2 \pi f t)$, with the field amplitude $H_{0}$ ranging from 5 to 100 Oe and the frequency $f$ in the gigahertz range, is supplied to the antenna, where $H_{0}$ is parallel to the $x$ - or $z$-axis. (In practical devices, a microwave voltage is loaded onto the antenna, where the accompanying rf Oersted field couples with the magnetization and excites spin waves). Near both ends of the waveguide, the damping parameter is increased to reduce the back reflection of spin waves ${ }^{8}$. Micromagnetic simulations were adopted to investigate the spinwave dynamics in these bended waveguides (See Methods section for details).

Figure 2 shows the snapshots of spin-wave distribution at a time after the steady state has been achieved. At low frequencies, the spin wave continuously follows the waveguide, with the wave vector fine
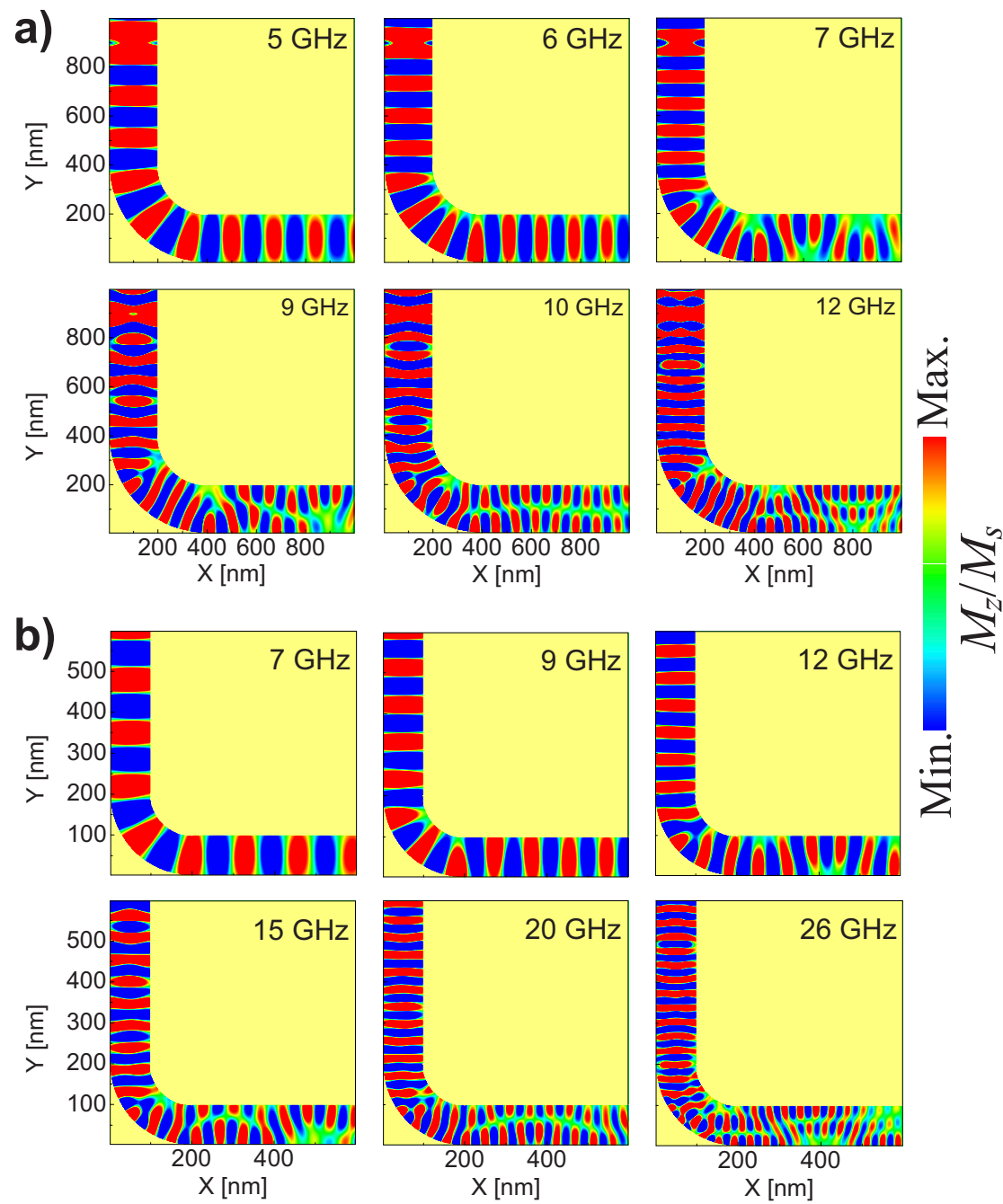

Figure $2 \mid$ Snapshots of spin-wave distribution inside the bended waveguides at a time of $\mathbf{1 0} \mathbf{n s}$ after applying the excitation field. The frequency values are labeled in each panel. The widths of the waveguide are (a) $200 \mathrm{~nm}$ and (b) $100 \mathrm{~nm}$, respectively. Note that the evolution of the spin-wave distributions as a function of the frequency exhibits the same tendency for the two different waveguides. 

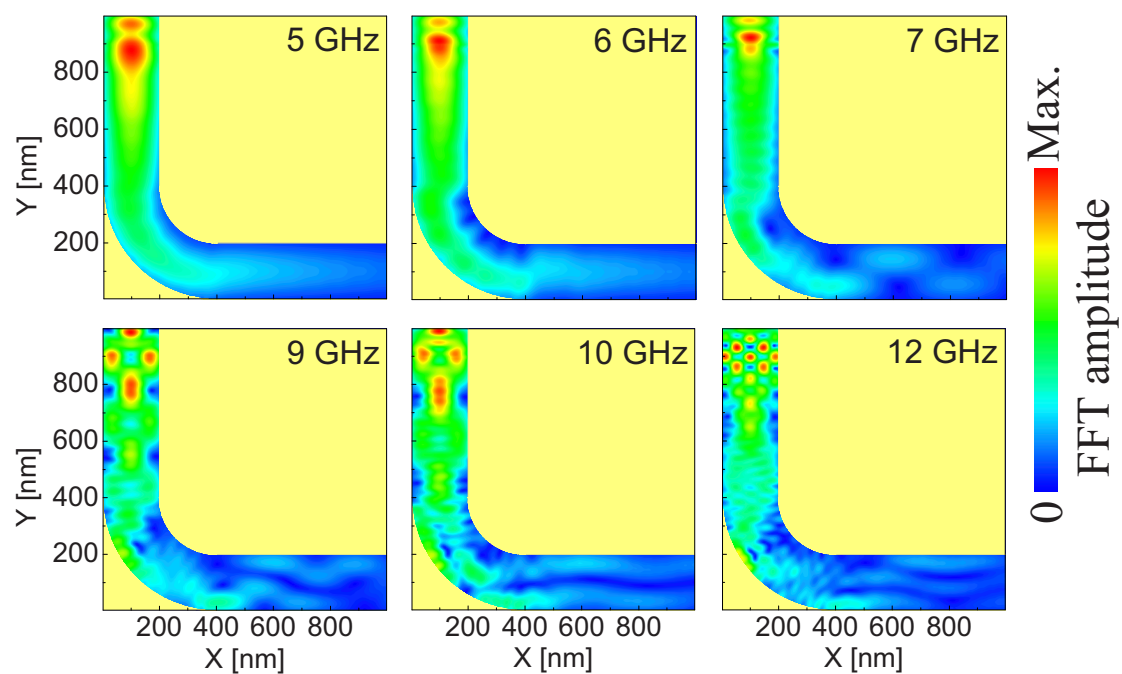

Figure 3 Fast Fourier transform (FFT) amplitude distributions for the spin waves corresponding to Fig. 2(a). The mode structures for spin waves at each frequency can be easily resolved, although the decay of spin waves with the propagation distance is not compensated.

adapting to the local magnetization ${ }^{7}$. The spin wave gone past the bend has the same spatial structure as that newly-radiated from the antenna, except for the decreased amplitude due to the intrinsic damping. At a slightly higher frequency, the scenario is substantially changed. Here, a zigzag-like propagation pattern is formed in the horizontal arm of the waveguide after the spin waves leaves from the bend, totally different from the continuous propagation picture for the spin wave before injection into the bend. This fact suggests that the spin wave does not always adapt to the waveguide. As the propagation manner of the spin wave changes from continuous to zigzag-like, the back reflection of the spin wave from the bend is enhanced. Actually, only for certain frequencies can the spin wave follow the waveguide without serious reflection loss. These findings reveal the limited transmission bandwidth of the spin-wave devices comprising folded components.

For the three lowest frequencies shown in Fig. 2(a) and 2(b), the spin waves before injection into the bend have the same feature of spatial distribution, say, they are nearly uniform across the waveguide width and continuous along the waveguide. The reduction of the spin-wave wavelength with the increase in the frequency arises from the dipole-exchange nature of the dispersion relation of the spin waves in such submicrometer waveguides ${ }^{15}$. When the spin wave gets into the bend, the curvature of the bend distorts the spatial profile of the spin wave. Subsequently, the spin-wave propagation behind the bend is affected.

As the frequency increases, the situation becomes more complex. At first, the symmetric width modes with higher order numbers are stimulated by the antenna to accompany the fundamental mode, leading to self-focusing ${ }^{16,17}$ of them in front of the bend. Next, the asymmetric modes are activated when the self-focused spin waves arrive at the bend. As a consequence, at least three branches of spin waves with different mode numbers coexist in the horizontal arm of the bended waveguide. The mutual interference among these spin waves produces fine mode pattern $s^{13}$. Remarkably, at particular frequency (e.g., $10 \mathrm{GHz}$ for the $200 \mathrm{~nm}$ wide waveguide and $20 \mathrm{GHz}$ for the $100 \mathrm{~nm}$ wide one), the beaten spin waves have very simple mode structure. Note that Figs. 2(a) and 2(b) correspond to two waveguides with different sizes. Thus, it is expected that the above stated discoveries represent a common feature of such submicron systems.

Figure 3 illustrates the Fourier amplitude maps of the spin waves with various frequencies propagating in a bended waveguide. The top panels correspond to these spin waves with the lowest frequencies, where the input antenna only excites the fundamental mode, and in addition the mode amplitude decreases with the propagation distance in front of the bend due to the natural damping of the spin wave. Inside the bended section, the amplitude distribution for the three spin waves with distinct frequencies differs substantially from each other. With the increase in the frequency, the spin-wave beam becomes narrower and meanwhile gets closer to the outer edge of the bend. It is also seen that the higher the frequency, the stronger the reflection of the incident beam at the entry of the bend, causing the spin-wave beam in front of the bend to present wavy borders at the frequency of $7 \mathrm{GHz}$. The bottom panels are for the spin waves with higher frequencies. Here, the mode patterns typical of selffocusing are clearly seen in the vertical arm. The transverse modulation of the beam strength complicates the spatial distribution of the spin-wave amplitude inside the bended section, and consequently the excitation of the secondary spin waves at the exit of the bend is equally intricate, so that the mode structure of those spin waves behind the bend is beyond the reach of the prediction.

\section{Discussion}

To see how the mode patterns are formed, the dispersion relation of these spin waves existing in the waveguide is derived, as plotted in Fig. 4. In the vertical arm before the bend, the modes with the order

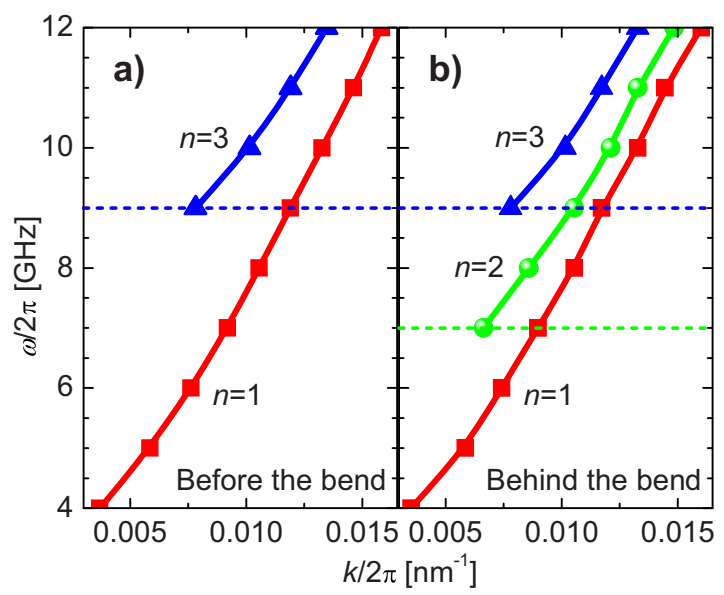

Figure $4 \mid$ Dispersion relation of the spin waves propagating in the $200 \mathrm{~nm}$ wide waveguide. Panels (a) and (b) are for the spin waves in the vertical and horizontal arms of the bended waveguide, respectively.

The mode numbers are marked next to each curve. 
number equal to 1 and 3 are present, as expected. These modes, being symmetric about the axis of the waveguide ${ }^{9,12}$, can be strongly coupled to the antenna's field and acquire the largest spectral weights. In the horizontal arm, the $2^{\text {nd }}$-order mode emerges in addition to the already existing symmetric modes, clearly indicating that this mode is excited due to the passage of the original spin waves through the bend.

Note that, the higher the mode number, the higher the frequency at which the related mode starts to occur. In the low-frequency range, only the fundamental mode is stimulated by the antenna, and no additional modes are excited when it goes through the bend and meanwhile the reflection of the original spin wave from the entry of the bend is negligible. As a result, the original spin wave accommodates to the whole waveguide, as seen in Ref. 7. In the mediumfrequency range, although still only the fundamental mode is driven by the antenna, the asymmetric $2^{\text {nd }}$-order mode is activated when the original spin wave flows into the bend, resulting in their coexistence in the horizontal arm of the waveguide. At the same time, the original spin wave is partially reflected from the bend's entry. As a consequence, the hybrid spin wave cannot always adapt to the waveguide with the wave vector parallel to the local magnetization. In the highfrequency range, more higher-order modes appear and cause multimode superposition, giving rise to finer interference patterns (see bottom panels of Figs. 2 and 3).

In the horizontal arm, the travelling waveguide modes can be formulated as follows,

$$
m_{n}(x, y, t)=C_{n} \sin \left(\frac{n \pi}{w^{*}} y\right) \cos \left(k_{x}^{n} x-\omega t-\varphi_{n}\right) \exp \left(-\frac{x}{D}\right),
$$

where the sine function describes the transverse profile of each mode, the cosine part the propagation character ${ }^{16}$, and the exponential part the spatial attenuation ${ }^{18}$. The parameter $w^{*}$ denotes the effective waveguide width ${ }^{19,20}$ for all the modes, $\omega$ the excitation frequency, $k_{x}^{n}$ the longitudinal wave number of the $n^{\text {th }}$-order mode, $\varphi^{n}$ the excitation phase, and $C_{n}$ the relative excitation efficiency. As reflected in Fig. 4 , for certain ranges of frequency, multiple modes with different order numbers exist simultaneously. These modes interfere mutually $^{13}$ and engender distinctive beating patterns (see Fig. 3). Averaging the module of the sum of $m_{n}$ over a large time interval $\tau$, one gets,

$$
A(x, y)=(1 / \tau) \int_{0}^{\tau}\left|\sum_{n} m_{n}(x, y, t)\right| d t,
$$

which depicts the spatial distribution of the total amplitude of the involved coherent modes ${ }^{16}$. The resulting theoretical mode patterns are in good agreement with those from micromagnetic simulations [as shown in Fig. 5(a)], proving that the zigzag-like patterns originate from the beating between the original symmetric modes and the reexcited asymmetric ones (See Methods for technical details).

For the lowest frequencies, the antenna simply sends out the fundamental-mode spin wave. When this spin wave reaches the bend, it has homogeneous phase and group velocity across the waveguide width [see top panels of Fig. 2(a) and 2(b)]. Subsequently, it sees different magnetization orientations at the inner and outer sides of the waveguide once entering the bend [see Fig. 5(b)]. Furthermore, the exchange field due to the curled magnetization is stronger at the inner side than it is at the outer side of the bend (see Ref. 21). The dispersion relation of the spin waves inside the bended section is accordingly changed compared to that in the outside. The altered dispersion relation distorts the spatial profile of the spin wave injected into the bend, and in turn creates the asymmetry of the dynamic field inside the bend, which makes the excitation of asymmetric modes possible. From the point of view of mode conversion, the bend simply plays the role of a kind of defect in a regular waveguide $^{22}$, which couples the symmetric modes radiated from the antenna to all the other modes of the waveguide available at a given
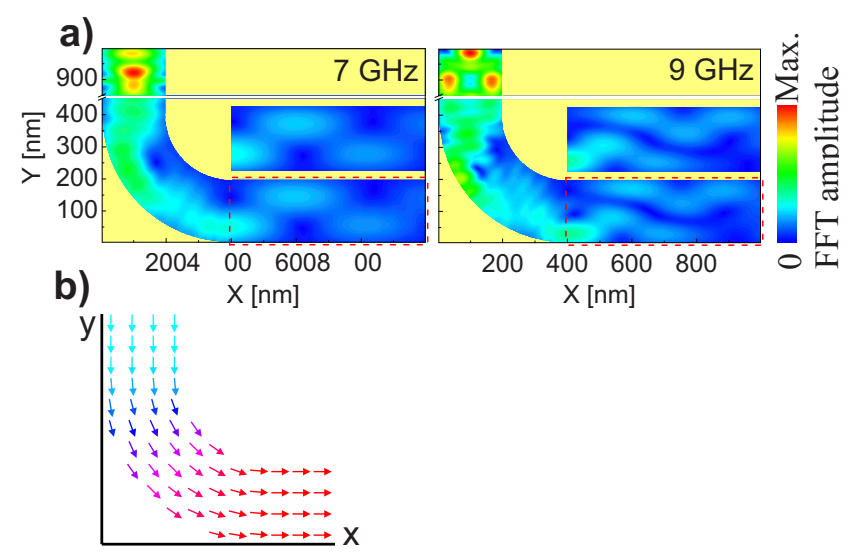

Figure 5 Comparison between theoretical and simulation results. (a) The exemplary mode patterns of the spin waves at the frequencies of 7 and $9 \mathrm{GHz}$ are presented. Enclosed in the boxes and inserted above are simulation and theoretical results, respectively. (b) Calculated groundstate configuration of magnetization around the bended section of the waveguide.

frequency. For these frequencies slightly higher than the turnon value, the spin-wave beam inside the bend spreads almost all over the waveguide width, so that the antisymmetric modes cannot be excited. Comparatively, for those higher frequencies, the beam entering the bend occupies only a portion of the waveguide width, inducing asymmetric distribution of the spin-wave amplitude, and thus is capable of driving the modes with the same symmetry. This explains why the spin waves with the lowest frequencies can continuously follow the waveguide, but those with higher frequencies can not (cf. top and bottom panels in Fig. 3).

Combining the bended section with a double-branch unit, a Mach-Zehnder type logic-NOT gate is formed [cf. Fig. 5(a)]. In this architecture, the antennae for inductive excitation and detection of the propagating spin waves are directly used as the input and output terminals of binary data. In real devices, the frequency of the excitation microwave is encoded as an input: $0 / 1$, with 0 being represented by a low-frequency and 1 by a high-frequency. The amplitude of the induced voltage is coded as an output: $0 / 1$, with 0 represented by a low-amplitude and 1 by a high-amplitude.

Micromagnetic simulations verify the functionality of the NOT gate [cf. Fig. 6(b)]. At the frequency of $6.8 \mathrm{GHz}$, the spin-wave beams injected into the double branches have a slight phase shift. When they converge in the right arm, constructive interference ${ }^{23,24}$ occurs and gives rise to high amplitude, i.e. logical 1 . At $10 \mathrm{GHz}$, the spin-wave beams ramified into the double branches retain a phase difference of $\sim \pi$, causing destructive interference ${ }^{20}$ of these spin waves in the collection strip and giving low amplitude, i.e. logical 0 . Note that the amplitude ratio of the two logic output signals is $m_{z}(1) / m_{z}(0)$ $\sim 3$, which can be further increased by optimizing pertinent factors. It is seen that the bend itself in the waveguide behaves as a control element in the gate, so that no external control modules ${ }^{1-5,25-28}$ are required any more, greatly simplifying the structure of the gate. Its operation is achieved solely by the bended waveguide as well as the excitation and probe antennae, efficiently improving the device speed by eliminating the external action cycle. We would like to point out that via frequency coding, the spin-wave filter developed by Kim et al. ${ }^{29}$ can directly realize a logic-NOT gate, which also does not need an extra control unit for operation, thus representing a simple and even more compact architecture.

In principle, arbitrary logic operations can be performed by using a logic-NOT gate and at least one additional two-input logical gate ${ }^{2,5,30}$. Several such integrated gates have been acquired in previous works by combining the basic NOT gates in a suitable manner ${ }^{2,3,5}$. 
a)

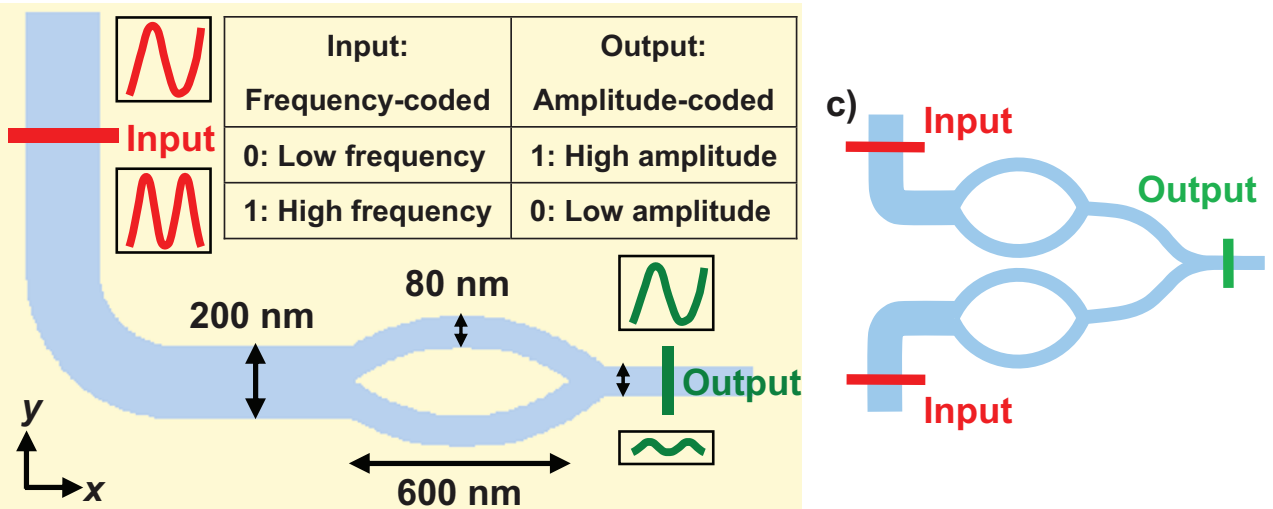

b)
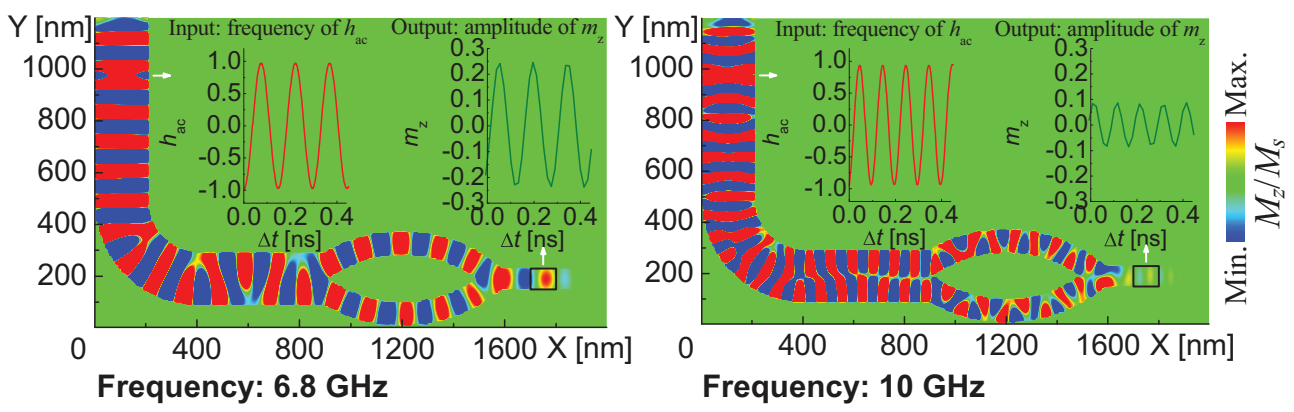

Figure 6 Demonstration of a logical NOT gate and schematic concept of a NAND gate based on such NOT gates. (a) Schematic diagram of the MachZehnder logic-NOT gate composed of a $200 \mathrm{~nm}$ wide bended waveguide. The sizes of the vertical arm and the bend are the same as given in Fig. 1 . The horizontal arm situated between the bend and the bifurcation is $500 \mathrm{~nm}$ in length. The double branches made of two $60^{\circ}$-arc-shaped strips are $600 \mathrm{~nm}$ long in the $x$-axis, and each of them is $80 \mathrm{~nm}$ wide. The strip connected to the double branches is $\sim 430 \mathrm{~nm}$ long and $80 \mathrm{~nm}$ wide. Each component of the gate is $5 \mathrm{~nm}$ thick. The red- and green-colored microstrips are data input and output terminals, respectively. Inset: truth table for the gate. (b) Snapshots of spin-wave distribution inside the gate for the frequencies of (left panel) $6.8 \mathrm{GHz}$ and (right panel) $10 \mathrm{GHz}$. Insets: each left subpanel shows the input signal loaded onto the radiation antenna, and each right subpanel plots the corresponding output signal detected by the reception antenna. If a low-frequency/high-frequency microwave signal (logic $0 / 1$ ) is sent into the waveguide via the input antenna, the output antenna will receive a highamplitude/low-amplitude spin-wave signal (logic 1/0) due to the constructive/destructive interference of the spin waves in the collection strip. (c) The logical NAND gate composed of two NOT gates. The output arms of these NOT gates are connected together, forming the single output terminal of the NAND gate. The spin-wave injection antennae are used as the input terminals.

Here, the same strategy is used to construct a logical NAND gate. In what follows, the detail is given. First, two identical NOT gates are fixed symmetrically about the $x$-axis, then their output arms are merged into a single collection arm to form the output terminal of the NAND gate, and finally the input antennae of the NOT units are directly used as the input terminals of the integrated gate, resulting in a Y-shaped structure of the logic-NAND gate, as shown in Fig. 6(c). Note that the spin-wave injection ends of our NAND circuit are not merged together, which is different from the situation in the seminal Ref. 2 where two Mach-Zehnder interferometric logic-NOT gates are connected in parallel to give a universal NAND gate. The reason is that for the frequency-coding scheme the spin waves flowing through individual NOT units of the NAND gate need to have different frequencies. The possibility of forming a NAND gate based on such NOT gates makes the bended magnonic waveguide very attractive as a building block for constructing practical logic devices.

In conclusion, we examine the spin-wave propagation in a bended magnetic waveguide on the submicrometer scale by means of micromagnetic simulations. The transmission of the spin waves through the bend is found to depend on the used frequency. For the lowest frequencies, the traveling spin waves continuously follow the entire waveguide $^{7}$. For the higher frequencies, the spin waves propagate along complex zigzag-like path $^{9}$ formed in the waveguide when they pass through the bended section. In the later case, the bend functions as a mode converter for the transmitting spin waves. By use of the frequency-dependent property, we numerically demonstrate the functionality of a Mach-Zehnder interferometric
logic-NOT gate based on the bended waveguide. We also suggest that it is possible to construct a logical NAND gate by combining such NOT gates ${ }^{2,5}$. These findings would be of importance for the design and optimization of magnonic devices involving bended waveguiding components.

\section{Methods}

Micromagnetic simulations. LLG micromagnetics simulator-a finite-difference $\operatorname{code}^{31}$, was adopted to numerically solve the Landau-Lifshitz-Gilbert equation-the equation of motion of magnetization ${ }^{32}$, by which the linear spin-wave dynamics ${ }^{33} \mathrm{can}$ be well described. The target waveguide was divided into a regular array of cubic meshes, which have a size of 5 or $2.5 \mathrm{~nm}$. The material parameters typical of Permalloy were used, i.e., the saturation magnetization is $860 \mathrm{emu} / \mathrm{cm}^{3}$, the exchange stiffness is $1.3 \times 10^{-6} \mathrm{erg} / \mathrm{cm}^{3}$, and the magnetocrystalline anisotropy was neglected. The Gilbert damping parameter is as high as 0.1 and 0.5 in the gray and blue end regions, respectively, and it is 0.01 in the region outside the absorbing ends. The equilibrium configuration of static magnetization in the bended waveguide under a zero external field is extracted by relaxing the system from artificially given states with saturated magnetization. In the ground state, the magnetization well follows the waveguide [see Fig. 5(b)] except in the end regions where the magnetization deviates from the long axis of the waveguide due to demagnetization.

A series of dynamic simulations using different values of the antenna width (5 to $60 \mathrm{~nm}$ ), the amplitude of excitation field $\left(H_{0}, 5\right.$ to $\left.100 \mathrm{Oe}\right)$, and the mesh size ( 2.5 or $5 \mathrm{~nm}$, both are smaller than the exchange length of Py) have been run to check the influence of these parameters on the transmission characteristics of the spin waves. Finally, no qualitative difference has been found among the simulation results. We thus estimate that the observed spin-wave dynamics well lie in the linear regime.

Experimentally, edge roughness in the real samples of a bended waveguide cannot be avoided for current microfabrication technologies. However, the real roughness can be numerically represented by the staircase-like edge steps along the curved boundary of a bended waveguide due to the rectangular mesh used in the finite-difference 
micromagnetic simulations (see Ref. 34). The fact that various simulations in our study with meshes of different sizes give the same results suggests that the edge roughness does not play a significant role for the addressed phenomenon.

Theory. In Eq. (1), we assume that all the spin waves have the same values of $D$ and $w^{*}$, regardless of the mode number and frequency. The value of $D$ is extracted by fitting the decay of the simulated FFT amplitude of the $5 \mathrm{GHz}$ spin wave behind the bend (the top-left panel in Fig. 3) against the propagation distance using an exponential function. The values of $k_{x}^{n}$ are derived from the simulated dispersion relation (Fig. 4) for the given frequencies $(\omega)$, and $w^{*}$ and $C_{n}$ are left as free parameters. It is found that the value of $w^{*}=240 \mathrm{~nm}$ makes the theoretica amplitude maps agree well with the simulated ones. For the frequency of $7 \mathrm{GHz}$, considering only the $1^{\text {st }}$ and $2^{\text {nd }}$ modes, one finds that $C_{1}=1, C_{2}=0.8, \varphi_{1}=0$, and $\varphi_{2}$ $=(15 / 180) \pi$ allows the best agreement between the theoretical and simulation results. For $9 \mathrm{GHz}$, the $3^{\text {rd }}$ mode is additionally taken into account, and then $C_{1}=1$, $C_{2}=1.2, C_{3}=0.8, \varphi_{1}=0, \varphi_{2}=\pi$, and $\varphi_{3}=(165 / 180) \pi$ leads to the best consistency. In the time-averaging procedure, the integration expressed by Eq. (2) is replaced by a summation, running over a time interval of $\tau=5 \mathrm{~ns}$ discretized into 1250 time steps (i.e., the step length is $4 \mathrm{ps}$ ), to mimic the finite-difference nature of the simulations ${ }^{31}$.

1. Hertel, R., Wulfhekel, W. \& Kirschner, J. Domain-wall induced phase shifts in spin waves. Phys. Rev. Lett. 93, 257202 (2004).

2. Lee, K.-S. \& Kim, S.-K. Conceptual design of spin wave logic gates based on a Mach-Zehnder-type spin wave interferometer for universal logic functions. J. Appl. Phys. 104, 053909 (2008).

3. Kostylev, M. P., Serga, A. A., Schneider, T., Leven, B. \& Hillebrands, B. Spin-wave logical gates. Appl. Phys. Lett. 87, 153501 (2005).

4. Schneider, T. et al. Realization of spin-wave logic gates. Appl. Phys. Lett. 92 022505 (2008)

5. Khitun, A., Bao, M. \& Wang, K. Spin wave magnetic nanofabric: A new approach to spin-based logic circuitry. IEEE Trans. Magn. 44, 2141-2152 (2008)

6. International Technology Roadmap for Semiconductors (ITRS) 2009 edition: Emerging Research Devices. (2009).

7. Dvornik, M., Au, Y. \& Kruglyak, V. V. Magnonics: Micromagnetic simulations in magnonics. Top. Appl. Phys. 125, 101-115 (2013).

8. Bance, S. et al. Micromagnetic calculation of spin wave propagation for magnetologic devices. J. Appl. Phys. 103, 07E735 (2008).

9. Clausen, P. et al. Mode conversion by symmetry breaking of propagating spin waves. Appl. Phys. Lett. 99, 162505 (2011).

10. Vogt, K. et al. Spin waves turning a corner. Appl. Phys. Lett. 101, 042410 (2012)

11. Vasiliev, S. V., Kruglyak, V. V., Sokolovskii, M. L. \& Kuchko, A. N. Spin wave interferometer employing a local nonuniformity of the effective magnetic field. J. Appl. Phys. 101, 113919 (2007).

12. Kostylev, M. P. et al. Dipole-exchange propagating spin-wave modes in metallic ferromagnetic stripes. Phys. Rev. B 76, 054422 (2007).

13. Büttner, O. et al. Mode beating of spin wave beams in ferrimagnetic $\mathrm{Lu}_{2.04} \mathrm{Bi}_{0.96} \mathrm{Fe}_{5} \mathrm{O}_{12}$ films. IEEE Trans. Magn. 34, 1381-1383 (1998).

14. Demokritov, S. O., Hillebrands, B. \& Slavin, A. N. Brillouin light scattering studies of confined spin waves: Linear and nonlinear confinement. Phys. Rep. $\mathbf{3 4 8}$ 441-489 (2001).

15. Choi, S. et al. Strong radiation of spin waves by core reversal of a magnetic vortex and their wave behaviors in magnetic nanowire waveguides. Phys. Rev. Lett. 98, 087205 (2007).

16. Demidov, V. E., Demokritov, S. O., Rott, K., Krzysteczko, P. \& Reiss, G. Mode interference and periodic self-focusing of spin waves in permalloy microstripes. Phys. Rev. B 77, 064406 (2008).

17. Demidov, V. E. et al. Nonlinear propagation of spin waves in microscopic magnetic stripes. Phys. Rev. Lett. 102, 177207 (2009).

18. Seo, S. M., Lee, K. J., Yang, H. \& Ono, T. Current-induced control of spin-wave attenuation. Phys. Rev. Lett. 102, 147202 (2009).
19. Guslienko, K. Y., Demokritov, S. O., Hillebrands, B. \& Slavin, A. N. Effective dipolar boundary conditions for dynamic magnetization in thin magnetic stripes. Phys. Rev. B 66, 132402 (2002).

20. Xing, X. J., Li, S. W., Huang, X. H. \& Wang, Z. G. Engineering spin-wave channels in submicrometer magnonic waveguides. AIP Adv. 3, 032144 (2013)

21. Tkachenko, V. S., Kuchko, A. N., Dvornik, M. \& Kruglyak, V. V. Propagation and scattering of spin waves in curved magnonic waveguides. Appl. Phys. Lett. 101, 152402 (2012).

22. Birt, D. R. et al. Diffraction of spin waves from a submicrometer-size defect in a microwaveguide. Appl. Phys. Lett. 95, 122510 (2009).

23. Podbielski, J., Giesen, F. \& Grundler, D. Spin-wave interference in microscopic rings. Phys. Rev. Lett. 96, 167207 (2006).

24. Choi, S., Lee, K.-S. \& Kim, S.-K. Spin-wave interference. Appl. Phys. Lett. 89, 062501 (2006).

25. Bayer, C., Schultheiss, H., Hillebrands, B. \& Stamps, R. L. Phase shift of spin waves traveling through a $180^{\circ}$ Bloch-domain wall. IEEE Trans. Magn. 41, 3094-3096 (2005).

26. Macke, S. \& Goll, D. Transmission and reflection of spin waves in the presence of Néel walls. J. Phys.: Conf. Ser. 200, 042015 (2010).

27. Hansen, U.-H., Demidov, V. E. \& Demokritov, S. O. Dual-function phase shifter for spin-wave logic applications. Appl. Phys. Lett. 94, 252502 (2009).

28. Demidov, V. E., Urazhdin, S. \& Demokritov, S. O. Control of spin-wave phase and wavelength by electric current on the microscopic scale. Appl. Phys. Lett. 95, 262509 (2009).

29. Kim, S.-K., Lee, K.-S. \& Han, D.-S. A gigahertz-range spin-wave filter composed of width-modulated nanostrip magnonic-crystal waveguides. Appl. Phys. Lett. 95, 082507 (2009).

30. Allwood, D. A. et al. Magnetic domain-wall logic. Science 309, 1688-1692 (2005).

31. Scheinfein, M. R. LLG Micromagnetics Simulator. (1997).

32. Gilbert, T. L. A phenomenological theory of damping in ferromagnetic materials. IEEE Trans. Magn. 40, 3443-3449 (2004).

33. Demidov, V. E., Demokritov, S. O., Rott, K., Krzysteczko, P. \& Reiss, G. Linear and nonlinear spin-wave dynamics in macro- and microscopic magnetic confined structures. J. Phys. D: Appl. Phys. 41, 164012 (2008).

34. Buda, L. D., Prejbeanu, I. L., Ebels, U. \& Ounadjela, K. Micromagnetic simulations of magnetization in circular cobalt dots. Comput. Mater. Sci. 24, 181-185 (2002).

\section{Acknowledgements}

This work is financially supported by the National Natural Science Foundation of China under the Grant No. 11104206 and by the Zhejiang Provincial Natural Science Foundation of China under the Grant No. LQ12A04002.

\section{Author contributions}

X.J.X. conceived and supervised the research. Y.L.Y. and X.H.H. helped perform the numerical simulations. S.W.L. made the theoretical analysis. All the authors discussed the results. X.J.X. and S.W.L. wrote the manuscript.

\section{Additional information}

Competing financial interests: The authors declare no competing financial interests.

How to cite this article: Xing, X.J., Yu, Y.L., Li, S.W. \& Huang, X.H. How do spin waves pass through a bend? Sci. Rep. 3, 2958; DOI:10.1038/srep02958 (2013).

(c) (i) (-) $\odot$ This work is licensed under a Creative Commons Attribution-

BY NG ND NonCommercial-NoDerivs 3.0 Unported license. To view a copy of this license, visit http://creativecommons.org/licenses/by-nc-nd/3.0 